\title{
Assessing Requirements for Agile Enterprise Architecture Management: A Multiple-Case Study
}

\author{
Philip Cammin \\ Institute of Information Systems \\ University of Hamburg \\ philip.cammin@uni-hamburg.de
}

\author{
Leonard Heilig \\ Institute of Information Systems \\ University of Hamburg \\ leonard.heilig@uni-hamburg.de
}

\author{
Stefan $\mathrm{Voß}$ \\ Institute of Information Systems \\ University of Hamburg \\ stefan.voss@uni-hamburg.de
}

\begin{abstract}
Agile enterprise architecture management (EAM) is a means to cope with the pressure for continuous architectural change in the context of short innovation cycles. However, the literature states a lack of practical EAM approaches. Therefore, this study aims at fostering the development of agile enterprise architecture frameworks (EAFs) by providing agile requirements and implementation concepts based on a literature review and an exploratory multiple-case study in three organizations of the logistics and construction tools industry. The multiple-case study's ranking by importance sets the focus on three requirements, for which implementation concepts are revealed. The gained insights make the concept and benefits of agile EAM tangible, indicate a shared understanding between the cases, and afford opportunities for future research.
\end{abstract}

\section{Introduction}

The digital transformation and rapidly changing market environments are the reason for organizations to adopt agile practices [1]. In the light of recent events, a good example for the necessity of agility can be observed in an exemplary organization within our multiple-case study. Although no restrictions concerning the at-door delivery of parcels within Germany have been imposed at the time of this writing, logistic companies rapidly adapted this crucial business process to enable contactless delivery and retain electronic proof of deliveries at the same time. Prior to the Coronavirus crisis, recipients signed the delivery on the carrier's handheld device by finger; now, recipients sign the delivery on the parcel with their own pen which is then photographed by the carrier. Self-organizing teams have implemented this change supported by the EAM function as a facilitator for bottom-up improvements, which literature often states as an agile attribute [2,3]. Moreover, the trend to adopt agile practices in organizations [1] is put into effect with the implementation of an agile EAM to mitigate the pressure of continuous delivery and improvements on a short-term basis [4]. According to Korolovych [5], agile EAM differs from traditional EAM in, e.g., an iterative and incremental development of the EA, the engagement with stakeholders and the focus on current and relevant organization needs. For the determination of agility, the agile manifesto has received attention in the past; for instance, it values "responding to change over following a plan" [6]. Kaddoumi and Watfa [7] adapted the agile manifestos principles [8] to fit into the context of EAM.

However, in well-known EAFs such as the Zachman Framework [9] or TOGAF [10], agile principles are not prevalent. The Zachman Framework lacks a methodology to apply agile principles, while TOGAF provides the Architecture Development Method (ADM) for guidance through its phases (prepare, develop, migrate and govern the EA, change management). ADM suggests early and incremental deployments of target EAs and early and frequent communication with stakeholders. Nevertheless, as a generic framework, it does not suggest implementations such as tools or concepts on how to develop "iterative[ly], over the whole process, between phases, and within phases" [10].

Agile practices, established in the field of software development $[1,11]$, receive interest for the application in EAM [12]. Agile development methods are the "de facto standard in large parts of many software organizations of different size," [13] and Scrum, as a popular generic agile process framework for "developing, delivering, and sustaining complex products" [14], could potentially be applied to TOGAF [12] to increase its agility. A process framework is reasonable because changing the EA is "not a once-off project" but rather a process taking into account an organization's continuously changing requirements [15]. Other researchers, e.g., [16], developed an agile approach for converting an EA which has been used in one organization after using big-bang approaches. About $94 \%$ of the organization's IT managers and enterprise architects (thirty and eight, respectively) 
would like to keep on working with an agile EAM approach. ${ }^{1}$

As early as 2009, Buckl et al. [17] conducted a report on the state-of-the-art in EAM. Regarding framework assistance, practitioners' opinions towards the practicability of EAFs can be summarized as being difficult. The authors state that "the frameworks appear theoretical and impossible to implement." Ten years later, Iyamu [15] states that "none of the EAFs seem to offer a step-by-step guidance from its theoretical concept to practice stage" and suggests research contributions towards "more concrete help and direction to practitioners and organizations from the standpoint of applicability and practice." A brief review of literature and practitioners' opinions by Kotusev reveals frameworks as being too theoretical, conceptual, complex and difficult to adopt; thus, being ignored or demoted to "idea contributors" in practice [18].

Recent related works in the area of agile EAM have been made by Hauder et al. [19] and Uludag et al. [20]. In the latter, the authors investigate the expectations of agile teams towards enterprise architects. Their multiple-case study indicates that enterprise architects are expected to provide technical guidance and support and that agile and lean methods can help in case of enterprise architects' high workload. Based on a literature review and the twelve agile principles, [19] put forward 33 principles, which were then empirically evaluated using a questionnaire answered by 105 industry experts, in which "the asked industry experts could only confirm or reject the application of an agile principle for EA management" [19]. The authors show that their proposed agile principles are adopted in a variety of industries and mention that "details about their actual implementation are yet to be revealed." Moreover, acknowledging the lack of EAM functions' uniformity in practice [21], further qualitative case studies could unearth working implementations and pitfalls of certain agile requirements, which could enhance an organization's EAM function and its support for agile teams. Against this background, the following research questions are formulated:

RQ1 What are potential requirements for agile EAM?

RQ2 How are these requirements implemented in practice?

Hereby, this study contributes to fill the important gap in understanding requirements for architectural agility and provides the necessary insights into the

\footnotetext{
$170.6 \%$ (Definitely agree), 23.5\% (Agree), 5,9\% (Partly agree) $0,0 \%$ (Do not agree), $0,0 \%$ (Definitely do not agree).
}

practical implementations in organizations outside of software development.

The remainder of the paper is structured as follows. In Section 2, a literature review is presented and requirements for agile EAM are put forward to answer RQ1. Subsequently, in Section 3, a multiple-case study in the logistics and construction tools domain is carried out to obtain insights into the practical implementations of the requirements to answer RQ2. Contrary to [19] who provide quantitative research on agile principles, this study obtains descriptive data and perceptions in a qualitative approach with a focus on implementations. Finally, the results are discussed and future research is proposed.

\section{Literature review}

The literature review utilized the keywords enterprise architecture, agile, scrum and framework for searches in the Web of Science and Google Scholar search engines. Beyond that, the pool of literature was enhanced with works already known to us and by conducting a backward and forward search. After reading through the abstracts, 43 works were selected for further investigation. In summary, 15 references were identified to consider agile principles (P1-P12 $\left.{ }^{2}\right)$ as useful in the context of EAM. These principles relate to the twelve agile principles adapted to the context of EAM in [7]. The use of the twelve agile principles seems justified concerning their granularity, while on the contrary, some terminologies are more abstract (see [22]). Most of the references are constructive research-oriented and propose a framework or a method. The references in this pool do not build upon each other, which is an indicator for a lack of standards in the area of agile EAM. Regarding the consideration of principles as valuable aspects of EAM, three particular principles (P1, P4 and P10) are suggested most often, which is reflected in the following subsections. Next, the findings are shown and agile requirements are put forward, which are then utilized in Section 3 to reveal implementations in practice.

\subsection{Continuous improvement}

The continuous delivery of architectural work (e.g., target EA planning or changing the EA in projects)

\footnotetext{
${ }^{2}$ Compressed version of P1-P12: 1: Early, continuous, valuable EA deliverables. 2: Welcome changing requirements. 3: Deliver working EA frequently. 4: EA stakeholders work together daily. 5: Motivate and trust individuals. 6: Face-to-face conversation. 7: Working EA is the primary measure of progress. 8: Sustainable EA development. 9: Continuous attention to technical excellence and good design. 10: Simplicity, the art of maximizing the amount of work not done, is essential. 11: Self-organizing teams. 12: Regular team reflection.
} 
can be understood as early and periodically (with the preference to a shorter time scale) improving the EA. Thereby, stakeholders are provided with an early empirical foundation to evaluate the impact of EAM in their organization. In this context, [23] identify the need for an early and periodically delivery of EA products to avoid a long period of non-visual benefits and dissatisfied information providers and [24] outline an EA management and development process considering the need of organizations for incremental EA development.

With continuous delivery, fast improvements of architectures can potentially be achieved (cf. [4]). Kaddoumi and Watfa [7] refer to [19] emphasizing that EA results are often outdated before they are complete and [23] state that EAM has to "continuously adapt to a volatile environment with changing criteria for goal fulfillment." Hanschke et al. [12] and Hensema [25] argue that new (stakeholder) requirements should be incorporated over time in the EA. A major aspect adopted in requirements (see R1-R8) is the continuous improvement of the EA. However, this requires to take into account recent and upcoming changes of internal stakeholder requirements (e.g., scope and level of detail of EA models) as well as external requirements (e.g., different laws in a multi-national organization). To this end, the practice of continuously monitoring such requirements in the first place becomes necessary.

R1 Valuable architectural work is realized early and periodically to improve the EA quickly.

R2 EAM should continuously monitor internal and external requirements to be able to react quickly.

\subsection{Scrum roles and prototypes}

Agile methods, including the generic process framework Scrum, are widely used in the field of software engineering [13]. Scrum has been investigated for the application in EAM (see, e.g., [12, 23]). Scrum roles such as the product owner and master could be considered for EAM. The product owner in Scrum is the single point to manage stakeholder requirements (e.g., the requirements of a customer in a software development project) through refining the product backlog [14]. In the context of EAM, [23] define the product owner to "represent the EA Stakeholders interested in gathering information about the EA," where the EA stakeholders are the customers. Frequently realized sprints comprise some of those requirements, which leads to continuous and valuable changes of the EA.

Another Scrum role is the Scrum master. In the context of EAM, the enterprise architect master ensures that the team of enterprise architects, who collect information about the EA, can always keep a clear focus if necessary information is not accessible (e.g., because of legal issues) [23]. Moreover, an enterprise architect master could advice the product owner on how to manage the product backlog and arrange meetings for the product owner and the team. For instance, a sprint retrospective's purpose is to improve the team performance, e.g., by discussing the last sprint concerning people and processes (cf. [14, 23]).

Prototypes are well-known means to test the feasibility of concepts. In this context, [16] suggest to develop prototypes that test co-existing systems "at data level" to minimize the risk of big-bang approaches. Once all requirements are fulfilled, the systems can be replaced and resources are released for subsequent EA projects. This could be of higher importance with critical components of the EA, such as an important business process or security-relevant infrastructures.

$\mathbf{R 3}$ The role EA owner is established as the single point to manage stakeholder requirements.

R4 The role enterprise architect master is established to guide the team of enterprise architects.

R5 Prototypes are used as approval gates before realizing a target EA.

\subsection{Simplicity}

Proper and Lankhorst [2] argue that agile enterprise architects should support the integration of bottom-up improvements in the "larger scheme of things" rather than to guide too much and in detail. An EA project should be assisted to "fit within the big picture" [2] and to be aligned with the enterprise objectives [3] rather than guiding a project on an overly-detailed level. In the same direction, [26] favors evolutionary problem solving over "extensively blueprinting the future rigidly" to facilitate collaboration and avoid overloading stakeholders.

The agile manifesto understands simplicity as to avoid unnecessary work or waste. Timm et al. [27] have created a framework for assessing the quality of EA models in which we deem the quality attributes usefulness and level of detail as well as complexity and documentation to be useful for guiding the team to avoid waste. Hensema [25] suggests an agile EA approach to restrict the development of EA artifacts to only essential ones and, as in Scrum [14], the definition of done is mentioned, which could be a means to raise the probability of a correct effort estimation and 
thus, to operate within fixed time-windows. Proper and Lankhorst [2] mention meticulously and overly-detailed architecture descriptions, where the term overly implies that waste is created.

In their agile approach to model an EA, [28] do not advocate or constrain to use certain modeling notations (e.g., bullet point lists, UML (Unified Modeling Language) models, BPMN (Business Process Model and Notation) processes and [16] suggest to keep EA artifacts "as simple as possible" to raise understandability for the stakeholders. Furthermore, the ease of use of models is essential once models are understood as "tools for thinking" [29]. These findings indicate that enterprise architects should commit to simplicity to avoid waste, which could be supported with an enterprise architect master (cf. [23]).

R6 Enterprise architects are committed to simplicity to avoid waste and additional effort.

\subsection{Communities}

Drews et al. [3] state that enterprise architects need to drive collaboration "across programs and teams for following a common technological vision." In some concepts such as the integration of EAM and agile software development teams [12] as well as BizDevOps teams [3], a shift of power from EAM to the teams is necessary. For instance, in BizDevOps the EAM role provides recommendations instead of guiding and refrains from "making and enforcing architectural decisions" with the EAM function fulfilling a "supporting and consulting role" [3].

As EAM assesses an organization holistically and supports interdisciplinary teams, this study agrees with [23], who suggest that an architect should have an interdisciplinary background. Jugel et al. [26] state that stakeholders from different areas, such as senior management and IT operations "take different perspectives on the EA" and, therefore, express different EA evolution concerns. If stakeholders' specific concerns towards EAM are not taken into account, the EAM acceptance rate decreases [23]. This could be further addressed with the establishment of an EA community, which "stimulates the exchange between people dealing with the EA" [12]. Moreover, collaboration can foster a better understanding and continuous improvement of architecture guidelines [30].

However, the collaboration with and within a multidisciplinary community presents several challenges. Buckl et al. [23] refer to [31], who is in line with Dreyfus [32], who states that stakeholder groups "utilize different languages and artifacts in their communication" which results in a mismatched communication. Kaddoumi and Watfa [7] refer to Ross [33], who suggests to use a single-page high-level EA graphic to "facilitate discussions between business and IT management" based on experiences in some case studies. This concept can foster the communication and a shared understanding among different stakeholder groups, team members and architects.

R7 An appropriate number of EA communities is in place for regularly stimulating the information exchange and collaboration of EA stakeholders.

R8 High-level single-page EA graphics are used to foster the discussions within EA communities.

To get insight about the relevance in practice, that is, how the requirements are implemented, a multiple-case study is presented in the following section.

\section{Case study}

The major means of this multiple-case study are interviews in three organizations that apply EAM. Rather than generalizing cases to population as in, e.g., quantitative surveys, this study aims to put forward analytic generalizations [34, 35], finding best practices for the implementation of the abstract requirements.

The interviews took place from May to June 2020 and were conducted in a semi-structured fashion. We used an interview guide with two main question groups (QG). The first QG addresses the participant's EAM function and perception of agile and non-agile EAM. The second QG seeks to gather details on the implementation of the identified requirements (R1-R8) (e.g., which concepts or tools are used?). We used supplementary internal EAM documentation (e.g., architecture principles, EA models) in our analysis to enable data triangulation to increase the credibility of findings [35].

The interviews were carried out with one of the enterprise architects of each organization (see Table 1); therefore, the term expert (interview) applies as "technical, process and interpretative knowledge" applied in a specific field [36], i.e., EAM, can be expected. Confronted with a time constraint, we focused on those requirements perceived as being most important by the participant. However, we did not restrict the participant to talk about less important requirements. This is reasoned, firstly, in the assumed interdependence of the requirements. Secondly, by allowing the participants to put emphasis "on those fields in which they were able to talk about concepts and experiences" the participants "were supposed to feel they were taken seriously as a competent partner" [37]. 
In advance of the interview, the participants received the aim and the procedure of the interview including the labels of the two QG as well as the eight requirements by email. In the beginning of the interview, its aim was briefly outlined again and the possibility for anonymity was clarified.

In this section, firstly, the organizations' EAM and their understanding of agile EAM are presented. Secondly, the implementations of requirements perceived as being most important by the organizations are addressed.

\subsection{EAM in brief}

The operational challenges of the EAM functions are the documentation and maintenance of the base EA model in collaboration with lead solution architects (ShipCo, ToolCo, LogCo) and to govern the EA in terms of aligned and established architectural guidelines (ToolCo). Furthermore, the consolidation of the IT application landscape (e.g., by identifying redundancies and decommissioning legacy systems) is highlighted. As a subsidiary company, ShipCo uses capability maps (a map of enabling combinations of EA components such as roles, processes and information [38]) to align with its parent company. In LogCo, the EAM function is attached to the IT function; therefore, EAM is traditionally a support function without influence on strategic business decisions. LogCo highlights the intermediary role of EAM by, e.g., supporting the exchange about project experiences between the stakeholders. On the strategic level, EAM defines a high-level target EA and measures to support the necessary transformation (to be reached within five years (ToolCo), respectively three to five years (ShipCo)). For example, ShipCo addresses the relevancy of cloud services, while in ToolCo, a current question concerns the relevancy and requirements of the integration of event-driven architectures (ToolCo). The results are passed to the lead solution architects who deal with different technological infrastructures and patterns, e.g., cloud and SAP integration. Subsequently, solution architects and software architects are working on specific projects and corresponding software application architectures (ToolCo).

All of the case organizations state to follow a "quite agile" EAM approach. ToolCo argues that "at least large projects" are consulted early and regularly in the development phase, e.g., in terms of established security architectural guidelines, instead of being consulted in the go-live phase. It is emphasized that "EAM is understood as a trainer rather than an arbiter," as alignment and collaboration between EAM and projects during the development phase are early and regularly undertaken, similar to frequent training sessions of a sports team. Similarly, $\operatorname{LogCo}$ advocates to provide support to projects as opposed to "forbidding solutions." Moreover, the importance of simple EA models is mentioned. The participants claim that the use of Scrum events such as Scrum retrospectives ( $\operatorname{LogCo}$ ), sprint planning and daily scrums enhances the agility of EAM (ShipCo and LogCo). Strategically, ShipCo's EAM joins yearly business-IT roadmap workshops (per business area) to discuss the business strategy development and necessary business-IT alignment on a high level; half-yearly shorter update workshops may be conducted. This leads to early architectural work on a conceptual level.

The meaning of the ranking by importance must be handled with caution because it was expressed by the participants spontaneously and given their abstract nature, the requirements could be understood as not being on the same hierarchy level and, therefore, difficult to compare (e.g., R6 could foster R1, i.e., avoiding additional effort could fasten the implementation of valuable architectural work). However, the ranking shows a tendency toward R1, $\mathrm{R} 6$ and $\mathrm{R} 8$ as these are ranked first place among all participants.

Subsequently, we focus on the requirements R1, R6 and R8 because of their ranking by importance (Table 1) and the responses of the participants. Table 2 summarizes the implementations by each case organization and the associations to the rationales.

\subsection{Implementations}

The requirement R1 ("Valuable architectural work is realized early and periodically to improve the EA quickly") is agreed by ToolCo as to govern projects to comply with the EAM's architectural vision, where agility in ToolCo leads to "at least large projects" being consulted early and regularly in the development phase. This usually means that architectural approval of EAM becomes "a simple administrative act." In contrast, experience shows that projects fail approval and are delayed, in case non-compliant (e.g., concerning security guidelines) planned architectures are discovered much later, e.g., near go-live.

Strategically, ShipCo understands early architectural work, as in, early aligning with business process owners, e.g., in half-yearly business-IT roadmap workshops. These workshops allow EAM to be aware of the business strategy development and to "know of potential requirements before these are formally raised." The 
Table 1. Case organizations.

\begin{tabular}{llllll}
\hline Code & $\begin{array}{l}\text { Location } \\
\text { of headquarter }\end{array}$ & $\begin{array}{l}\text { Employees } \\
\text { globally (c.) }\end{array}$ & Industry & $\begin{array}{l}\text { Enterprise } \\
\text { architects }\end{array}$ & $\begin{array}{l}\text { Ranking of requirements by } \\
\text { importance }\end{array}$ \\
\hline ToolCo & Europe & $25.000+$ & Construction tools & 2 & R6 $>$ R1 $>$ R2 $>$ [R4, R7, R8] $>$ R3 \\
ShipCo & Germany & $5.000+$ & Logistics & 2 & {$[\mathrm{R} 1, \mathrm{R} 8]>[\mathrm{R} 6, \mathrm{R} 2, \mathrm{R} 7]>\mathrm{R} 3$} \\
LogCo & Germany & $10.000+$ & Logistics & 2 & $\mathrm{R} 6>[\mathrm{R} 7, \mathrm{R} 8]>\mathrm{R} 1>\mathrm{R} 2>\mathrm{R} 5$ \\
\hline
\end{tabular}

1 The square bracket signals the same rank among the enclosed requirements.

workshops have two major aims. Firstly, to ShipCo, these workshops are critical, as business stakeholders tend to formally raise concrete IT requirements short to deadline. The workshops allow to establish a common picture on the development direction of the business, followed by discussions on how the IT should develop in general to support the business, e.g., with cloud computing. Subsequently, EAM could then investigate the feasibility and preparation for the integration of potential new EA components early on. Secondly, the early involvement allows EAM to influence the shape of business products. For instance, if the business' IT requirements oppose the high-level target EA, ShipCo's EAM supports the business in finding an acceptable trade-off between following and changing the target EA.

ShipCo sees periodical alignment with the business as critical and states: "You never reach the target EA since the requirements always change." For the planning of EA work, in ShipCo and LogCo, Scrum is partially applied. Short-term sprints are means of agile planning and incremental, periodical development of the target EA, and methods, such as the retrospective, are applied to improve the team performance. However, both organizations reject the Scrum role EA owner (R3). The case organizations' EAM functions own a supporting role for the organizations' managements and projects; the concept of a single entity to represent these EA stakeholders is not applied. EAM's supporting role also manifests in the irregular meeting of different communities, which can be used to explain and offer the support of EAM to business and IT people (ShipCo, LogCo). Given the supporting role of EAM itself and the few enterprise architects in each organization, the benefit of the role enterprise architect master (R4) is not recognized by the participants.

The rationales of the requirement R6 ("Enterprise architects are committed to simplicity to avoid waste and additional effort') can be divided into two areas. Simplicity plays a major role in the documentation of the system landscape in high-level EA models using commercial EAM tools (e.g., based on the ADOxx (see
[39]) meta-modeling platform). All case organizations utilize meta models to maintain simplicity of EA models. For instance, a whitelist of attributes (e.g., multi-factor authentication necessity for a system) is established based on the "most important requirements" within the community of top EA model stakeholders (ToolCo). Overly-detailed EA models are not necessary, e.g., by adding concrete database table names. This does not increase the business value of EAM as a strategic and holistic discipline. Such information can be found in other sources (e.g., application documentation) available to the relevant actors (e.g., software architects and engineers) as stated by ToolCo.

ToolCo states that the continuous effort to maintain the integration of high-level EA models and data models for EA model stakeholders cannot be justified. Because of maintenance and complexity issues, ShipCo and LogCo have recreated their entire EA model based on a simple meta model with much fewer entities. However, finding a trade-off between detailed and simple meta models is a challenge. For instance, in LogCo, the generalization of the entities platform, application and microservice into a single entity type application was reversed. Regarding strategic target modeling, ShipCo emphasizes that the level of detail should decrease with a longer planning horizon. This is reasoned with a higher uncertainty of detailed EA target-models, which is often criticized by EA target-model stakeholders. Subsequently, this could lead to a plan that creates dependencies on uncertain detailed EA target-models, which are difficult to adapt (and correct) later. This is especially true for target-modeling in strategic workshops, such as business-IT roadmap workshops, as these do not reveal sufficiently detailed information (ShipCo).

The governance of a simple EA can be exemplified based on a current project in ToolCo, i.e., the implementation of a new cloud-based customer-relationship-management (CRM) platform. The aim of the platform is to avoid the development and integration of self-made individual solutions and to 
promote the use of standard solutions. The platform is updated several times a year by the provider. Deviations from the standard, introduced by ToolCo, increase complexity, incompatibility and maintenance effort which generates costs. Compatibility to future updates is a major concern to be able to profit from innovative updates of the provider (or from third parties) without the need to modify EA components by own means, an "essential reason to use a cloud-based platform." ToolCo's EAM considered cloud-based CRM platforms because those fit in the overall goal to "maximize simplicity." An architectural principle in ToolCo that strengthens simplicity is that "a business requirement should only be implemented once." To support projects, EAM joins a three-weekly code review. LogCo even states that "modern enterprise architects should not consider themselves too good for looking at source code once in a while." It is added that in the context of project support, EAM is a central point of contact that incorporates the knowledge of many projects and can, therefore, provide certain guidance (on-demand) to project teams.

In general, ToolCo's EAM has established 13 architectural principles, for which an abstract formulation is chosen deliberately. This is based on the experience that concrete principles can become outdated (fast) and differ per jurisdiction, thus, continuously generating additional effort. For instance, the abstract principle "applicable laws and data governance guidelines have to be accepted" takes into account regulations such as the General Data Protection Regulation (GDPR) in the European Union [40]). Article 97 (5) of the GDPR asks the EU Commission to propose amendments to consider progress in information technology and information society if necessary. More concrete principles would need to reflect these changes, which is avoided.

All participants agree with the concept of communities (see R7) being an important tool to share information on the EA. Such horizontal communities (e.g., meeting of all IT managers) and vertical communities (e.g., multi-project-related yearly roadmap business-IT workshops or single-project-related workshops joined by business process owners, IT management, enterprise architects) require high-level EA graphics that convey complex EAs in a fast and comprehensive way, because for the majority of stakeholders, EA models are not daily business. Therefore, the implementation of the requirement $\mathbf{R 8}$ ("High-level single-page EA graphics are used to foster the discussions within EA communities") is realized. These graphics can be understood as simplified models that do not follow a defined EA meta model.
For instance, ShipCo describes high-level EA graphics to depict the application landscape or a uniform terminology as a crucial tool. These allow communities to have a common understanding as well as to establish concise terms which facilitate the communication on a daily basis. These graphics, in comparison to large documents and comprehensive EA models, are easier to be accessed on a daily basis (i.e., printed and exhibited at a wall) and to be internalized. LogCo emphasizes that EAM produces many graphics (compared to formal EA models) to increase the usability, and therefore, business stakeholders' acceptance. These graphics are not only used in established communities but also in meetings between single stakeholders. For instance, the chief information officer and an enterprise architect discuss the criticality, technical abilities or the resilience degree of applications as part of the technology and risk management.

\section{Discussion}

The literature states a lack of practical EAM approaches as frameworks are considered generic and complicated $[15,18] . \quad$ It is suggested to reveal implementations for agile EAM [19]. In a mixed-methods research design, eight requirements are put forward by means of a literature review. Subsequently, an exploratory multiple-case study in organizations of the logistics and construction tools industry is carried out. An importance ranking of the requirements is presented, followed by an assessment of the three most important requirements as an instrument to obtain industry insights and best practices.

The case organizations are observed to follow a governance model that supports self-organizing teams, independent of the case industry. The supporting and consulting role promotes fast bottom-up architecture improvements, and synergies among teams by effective regular and early communication (see R1). This study indicates that higher level target EAs are valued for allowing projects enough decision-making scope within a "normative enterprise architecture" [2], and to make use of innovative applications and technologies rather than to "forbid," in line with [5]. This way bottom-up improvements could contribute to the digital transformation which is considered to include "the exploration of digital technologies to improve existing processes, and the exploration of digital innovation, which can potentially transform the business model" [41]. The supporting role also means that agile EAM acts as a facilitator for collaboration across different projects, not only to achieve a "common technological vision" [3], but also to share pitfalls 
Table 2. Summary of the multiple-case study findings.

\begin{tabular}{|c|c|c|c|c|}
\hline Alternative rationales & Implementations & ToolCo & ShipCo & $\log \mathrm{Co}$ \\
\hline \multicolumn{5}{|c|}{$\boldsymbol{R} \boldsymbol{1}$ Valuable architectural work is realized early and periodically to improve the EA quickly. } \\
\hline $\begin{array}{l}\text { a. To be aware of potential IT requirements from } \\
\text { business and to prepare the implementation ahead } \\
\text { of time. }\end{array}$ & $\begin{array}{l}\text { Present EA and offer EAM support in irregular } \\
\text { communities. }\end{array}$ & & a & a \\
\hline $\begin{array}{l}\text { b. To find the trade-off, to negotiate the high-level } \\
\text { target EA. }\end{array}$ & Half-yearly business-IT roadmap workshops. & & $\mathrm{a}, \mathrm{b}$ & \\
\hline c. To avoid delay of projects. & Large projects are consulted early. & $\mathrm{c}, \mathrm{d}$ & & \\
\hline \multirow{2}{*}{$\begin{array}{l}\text { d. To constrain projects to architectural boundaries } \\
\text { early on. }\end{array}$} & Scrum sprint planning and daily Scrum & & $\mathrm{d}$ & $\mathrm{d}$ \\
\hline & Scrum retrospective. & & & $\mathrm{d}$ \\
\hline \multicolumn{5}{|c|}{$\boldsymbol{R 6}$ Enterprise architects are committed to simplicity to avoid waste and additional effort. } \\
\hline e. To enable uniform models. & Meta-modeling. & e & $\mathrm{e}, \mathrm{h}$ & $\mathrm{e}, \mathrm{h}$ \\
\hline $\begin{array}{l}\text { f. To avoid overly-detailed models that become } \\
\text { outdated fast. }\end{array}$ & $\begin{array}{l}\text { Attribute whitelist and entities in meta models } \\
\text { are governed. }\end{array}$ & $\mathrm{f}, \mathrm{g}, \mathrm{h}$ & & $\mathrm{f}$ \\
\hline $\begin{array}{l}\text { g. To avoid the integration of high-level EA } \\
\text { models and data models. }\end{array}$ & Principle "Target models are less detailed." & & $\mathrm{f}, \mathrm{h}$ & \\
\hline \multicolumn{5}{|l|}{ h. To reduce model maintenance effort. } \\
\hline \multirow[t]{2}{*}{$\begin{array}{l}\text { i. To avoid the development, integration and } \\
\text { maintenance of self-made individual solutions. }\end{array}$} & $\begin{array}{l}\text { Abstract architectural principles, e.g., } \\
\text { preference to standardized cloud-based } \\
\text { solutions. }\end{array}$ & $\mathrm{i}$ & & \\
\hline & Conduct code reviews. & $\mathrm{i}$ & & $\mathrm{i}$ \\
\hline
\end{tabular}

R8 High-level single-page EA graphics are used to foster the discussions within EA communities.

j. To facilitate (community) discussions and Informal and user-oriented models or graphics. increase common understanding.

$\mathrm{j}, \mathrm{k} \quad \mathrm{j}, \mathrm{k}$

k. To increase usability of models on a daily basis, Uniform terminology. and acceptance of EAM.

$\mathrm{j}, \mathrm{k} \quad \mathrm{j}, \mathrm{k}$

experienced in past projects as outlined by the case organizations. These patterns are strengthened by the fact that the requirement $\mathrm{R} 3$ (establish role $E A$ owner) is not perceived important in all cases. The importance ranking and implementations indicate where research could improve existing practices. However, it is noteworthy that outlining the rationales and implementations (Table 2) reveals how those intertwine, e.g., both a uniform terminology and model could facilitate the consultation of projects. Thereby, an integrated approach (combination of implementations) is useful for further research, which also considers the different needs of stakeholders (e.g., in vertical communities), and a hierarchy of requirements. The cases also show that organizations understand to differentiate the concept of simplicity, and apply it on multiple aspects, e.g., artifacts such as models (see, e.g., "keep enterprise architecture artifacts as simple as possible" [16]), and governance (e.g., trend to use standardized cloud services also identified in [42]). Two cases have failed on the artifact aspect in finding the right trade-off, either having too detailed or too simple meta models, and thus, relaunched their meta models. This indicates potential research opportunities to support organizations in determining the correct level of detail for (meta-) models and to support transitions where it is necessary (e.g., in case of changing needs), taking into account potential dependencies on other implementations. Moreover, it is noteworthy that the cases ignore the potential of enterprise modeling to facilitate strategic business-IT workshops (see, e.g., 4EM [43, 44]).

Research and practice have both put forward and adapted EAFs (adaption is a crucial step put forward in EAFs themselves (e.g., in TOGAF [10])) to accommodate changing needs over time, but practicability remains an issue. This qualitative research unearthed why the presented implementations work for the assessed organizations, and what adjustments were necessary to fulfill certain agile requirements, which can be used to generalize to similar characterized organizations. Globally operating EAM functions, 
such as ToolCo's, may see it fit to define abstract architecture principles, e.g., regarding data protection regulation, to reduce continuous administrative effort; EAM functions of subsidiary companies, such as ShipCo, may have a strong need to align with their parent companies utilizing capability maps, whereas these are not pursuit in LogCo. Therefore, it can be considered ambitious to find a domain, to which sets of those requirements and implementations can be generalized. However, discussing the requirements and their importance, rationales as well as the organizational fit of implementations, put forward in this paper, could enable organizations to achieve a homogeneous understanding of EAM (i.e., its meaning, purpose and scope [45]), and therefore, foster organizations' EAM functions.

This paper contributes to a better understanding of important aspects of agility. It lays the focus on three requirements perceived as being most important by the case organizations and thus, does not discuss all of the requirements put forward in the literature. The potential implications may stimulate further research to unveil additional rationales and implementations, and their interplay, in both quantitative and qualitative research.

\section{References}

[1] D. Gerster, C. Dremel, W. Brenner, and P. Kelker, "How Enterprises Adopt Agile Forms of Organizational Design: A Multiple-Case Study," ACM SIGMIS Database, vol. 51, pp. 84-103, 2020.

[2] H. A. Proper and M. M. Lankhorst, "Enterprise Architecture - Towards Essential Sensemaking," Enterprise Modelling and Information Systems Architectures, vol. 9, no. 1, pp. 5-21, 2014.

[3] P. Drews, I. Schirmer, B. Horlach, and C. Tekaat, "Bimodal Enterprise Architecture Management: The Emergence of a New EAM Function for a BizDevOps-Based Fast IT," in Proceedings of the 21st International Enterprise Distributed Object Computing Conference workshops (EDOCW). Piscataway, NJ: IEEE, 2017, pp. 57-64.

[4] X. Yu, Y. Cui, and H. Wang, "Research on an Agile Enterprise Architecture Design Method," in Proceedings of the 4th International Conference on Electrical \& Electronics Engineering and Computer Science (ICEEECS). Paris, France: Atlantis Press, 2016, pp. 246-249.

[5] P. Korolovych, "A new approach to enterprise architecture to meet business needs," 2019. Available: https://www.ibm.com/ua-uk/events/think-summit/ assets/pdf/Think_2019_EA_KorolovychPP.pdf (Accessed 11/27/2019).

[6] K. Beck, M. Beedle, A. van Bennekum, A. Cockburn, W. Cunningham, M. Fowler, J. Grenning, J. Highsmith, A. Hunt, R. Jeffries, J. Kern, B. Marick, R. C. Martin, S. Mellor, K. Schwaber, J. Sutherland, and D. Thomas, "Manifesto for Agile Software Development," 2001. Available: https://agilemanifesto. org/ (Accessed 5/22/2019).
[7] T. Kaddoumi and M. Watfa, "A Proposed Agile Enterprise Architecture Framework," in Proceedings of the 6th International Conference on Innovative Computing Technology (INTECH). IEEE, 2016, pp. $52-57$.

[8] K. Beck, M. Beedle, A. van Bennekum, A. Cockburn, W. Cunningham, M. Fowler, J. Grenning, J. Highsmith, A. Hunt, R. Jeffries, J. Kern, B. Marick, R. C. Martin, S. Mellor, K. Schwaber, J. Sutherland, and D. Thomas, "Principles behind the Agile Manifesto," 2001. Available: https://agilemanifesto.org/principles. html (Accessed 5/22/2019).

[9] J. A. Zachman, "A Framework for Information Systems Architecture," IBM Systems Journal, vol. 26, no. 3, pp. 276-292, 1987.

[10] The Open Group, The TOGAF Standard, Version 9.2, 2018.

[11] G. Lee and W. Xia, "Toward Agile: An Integrated Analysis of Quantitative and Qualitative Field Data on Software Development Agility," MIS Quarterly, vol. 34, pp. 87-114, 2010

[12] S. Hanschke, J. Ernsting, and H. Kuchen, "Integrating Agile Software Development and Enterprise Architecture Management," in Proceedings of the 48th Hawaii International Conference on System Sciences (HICSS). Piscataway, NJ: IEEE, 2015, pp. 4099-4108.

[13] A. Scheerer, T. Hildenbrand, and T. Kude, "Coordination in Large-Scale Agile Software Development: A Multiteam Systems Perspective," in Proceedings of the 47th Hawaii International Conference on System Sciences, (HICSS). IEEE Computer Society, 2014, pp. 4780-4788.

[14] K. Schwaber and J. Sutherland, "The Scrum Guide," 2017. Available: https://www.scrumguides.org/ scrum-guide.html (Accessed 5/22/2019).

[15] T. Iyamu, "What Are the Implications of Theorizing the Enterprise Architecture?" Journal of Enterprise Transformation, vol. 15, no. 3, pp. 1-22, 2019.

[16] O. E. Balcicek, M. Gundebahar, and S. Cekerekli, "An Agile Approach for Converting Enterprise Architectures," in Proceedings of the 1st International Conference on Technological Advances in Electrical, Electronics and Computer Engineering (TAEECE). IEEE, 2013, pp. 380-386.

[17] S. Buckl, A. M. Ernst, J. Lankes, F. Matthes, and C. M. Schweda, "State of the Art in Enterprise Architecture Management 2009." Available: https://wwwmatthes. in.tum.de/file/1wbr6a65ggqkx/Sebis-Public-Website/ Publications/Bu09h.pdf (Accessed 5/14/2019).

[18] S. Kotusev, "Critical Questions in Enterprise Architecture Research," International Journal of Enterprise Information Systems, vol. 13, no. 2, pp. 50-62, 2017.

[19] M. Hauder, S. Roth, C. Schulz, and F. Matthes, "Agile Enterprise Architecture Management: An Analysis on the Application of Agile Principles," in Proceedings of the 4th International Symposium on Business Modeling and Software Design (BMSD). SciTePress, 2014, pp. $38-46$

[20] Ö. Uludag, M. Kleehaus, N. Reiter, and F. Matthes, "What to Expect from Enterprise Architects in Large-Scale Agile Development? A Multiple-Case Study," in 25th Americas Conference on Information Systems (AMCIS). AIS, 2019. 
[21] S. K. Muthukaruppan and V. Nandey, "Executive summary," 2019. Available: https://www.cognizant.com/whitepapers/ creating-an-agile-enterprise-architecture-codex 4617 pdf (Accessed 2/10/2020).

[22] A. Q. Gill, "Towards the Development of an Adaptive Enterprise Service System Model," in Proceedings of the 19th Americas Conference on Information Systems (AMCIS). AIS, 2013, pp. 3669-3677.

[23] S. Buckl, F. Matthes, I. Monahov, S. Roth, C. Schulz, and C. M. Schweda, "Towards an Agile Design of the Enterprise Architecture Management Function," in Proceedings of the 15th International Enterprise Distributed Object Computing Conference Workshops $(E D O C W)$. Los Alamitos, CA: IEEE, 2011, pp. 322-329.

[24] M. Pulkkinen and A. Hirvonen, "EA Planning, Development and Management Process for Agile Enterprise Development," in Proceedings of the 38th Annual Hawaii International Conference on System Sciences (HICSS). IEEE, 2005.

[25] M. Hensema, "Applying Agile in Enterprise Architecture." Available: https://essay.utwente.nl/68228/ 1/Hensema_MA_EEMCS.pdf (Accessed 5/7/2019).

[26] D. Jugel, C. M. Schweda, and A. Zimmermann, "Modeling Decisions for Collaborative Enterprise Architecture Engineering," in Proceedings of the 27th Conference on Advanced Information Systems Engineering Workshops (CAiSE). Cham: Springer, 2015, vol. 215, pp. 351-362.

[27] F. Timm, S. Hacks, F. Thiede, and D. Hintzpeter, "Towards a Quality Framework for Enterprise Architecture Models," in Proceedings of the 5th International Workshop on Quantitative Approaches to Software Quality (QuASoQ). CEUR-WS, 2017, pp. $14-21$.

[28] F. Gout and P. Robinson, "XAF: A Minimalist EA Framework for an Agile Environment," Cutter IT Journal, vol. 19, no. 3, pp. 16-25, 2006.

[29] M. Pidd, Tools for Thinking: Modelling in Management Science. Chichester, England: Wiley, 2003.

[30] Ö. Uludag, S. Nägele, and M. Hauder, "Establishing Architecture Guidelines in Large-Scale Agile Development Through Institutional Pressures: A Single-Case Study," in Proceedings of the 25th Americas Conference on Information Systems (AMCIS). AIS, 2019.

[31] M. Lankhorst, Enterprise Architecture at Work. Heidelberg: Springer, 2009.

[32] D. Dreyfus, "Information System Architecture: Toward a Distributed Cognition Perspective," in Proceedings of the International Conference on Information Systems (ICIS). AIS, 2007, paper 131

[33] J. W. Ross, "Enterprise Architecture: Depicting a Vision of the Firm," CISR Research Briefings, vol. IV, no. 1B, pp. 1-3, 2014. Available: ftp://ftp.software. $\mathrm{ibm} . c 0 m /$ la/documents/imc/la/pe/news/events/mit_2010/ 3a_2004_03_1b_entarchvisfirm.pdf (Accessed 5/3/2019).

[34] M. D. Myers, Qualitative Research in Business \& Management. London: Sage, 2013.

[35] R. K. Yin, Case study research and applications: Design and methods. Los Angeles: Sage, 2018.
[36] A. Bogner and W. Menz, "The theory-generating expert interview: Epistemological interest, forms of knowledge, interaction," in Interviewing Experts, ser. Research methods series. Basingstoke: Palgrave Macmillan, 2009, pp. 43-80.

[37] G. B. Christmann, "Expert Interviews on the Telephone: A Difficult Undertaking," in Interviewing Experts, ser. Research methods series. Basingstoke: Palgrave Macmillan, 2009, pp. 157-183.

[38] J. Zdravkovic, J. Stirna, and J. Grabis, "Capability Consideration in Business and Enterprise Architecture Frameworks," in Capability Management in Digital Enterprises. Cham: Springer International Publishing, 2018, pp. 41-56.

[39] D. Karagiannis, M. Lee, and R. A. Buchmann, "Using Metamodeling for Requirements Engineering: A Best-Practice with ADOxx," in 27th International Requirements Engineering Conference (RE). IEEE, 2019, pp. 498-499.

[40] European Parliament, "Regulation (EU) 2016/679," 2016. Available: https://eur-lex.europa.eu/legal-content/ EN/TXT/PDF/?uri=CELEX:32016R0679\&from=EN

[41] S. Berghaus and A. Back, "Stages in Digital Business Transformation: Results of an Empirical Maturity Study," in 10th Mediterranean Conference on Information Systems (MCIS). AIS, 2016, paper 22.

[42] S. H. Kaisler and F. Armour, "15 Years of Enterprise Architecting at HICSS: Revisiting the Critical Problems," in 50th Hawaii International Conference on System Sciences (HICSS). AIS, 2017, pp. 1-10.

[43] K. Sandkuhl, J. Stirna, A. Persson, and M. Wißotzki, Enterprise Modeling. Berlin: Springer, 2014.

[44] J. Stirna and A. Persson, Enterprise modeling. Cham: Springer, 2018.

[45] S. Bean, "Re-thinking Enterprise Architecture using Systems and Complexity Approaches," Journal of Enterprise Architecture, vol. 6, no. 4, pp. 7-13, 2010. 